Mohamad: New Analysis to Measure the Capacitance and Conductance

\title{
New Analysis to Measure the Capacitance and Conductance of
}

\section{MOS Structure}

\author{
Dr. W. F. Mohamad \& Dr. L. S. Ali \\ Electrical Engineering Department \\ College of Engineering \\ University of Mosul
}

\begin{abstract}
:
In this research thin film layers have been prepared at alternate layers of resistive and dielectric deposited on appropriate substrates to form four - terminal $R$ Y-NR network. If the gate of the MOS structures deposited as a strip of resistor film like NiCr, the MOS structure can be analyzed as R-Y-NR network. A method of analysis has been proposed to measure the shunt capacitance and the shunt conductance of certain MOS samples. Matlab program has been used to compute shunt capacitance and shunt conductance at different frequencies. The results computed by this method have been compared with the results obtained by LCR meter method and showed perfect coincident with each other.
\end{abstract}

Keywords: Thin Film R-Y-NR Network; MOS R-Y-NR Network

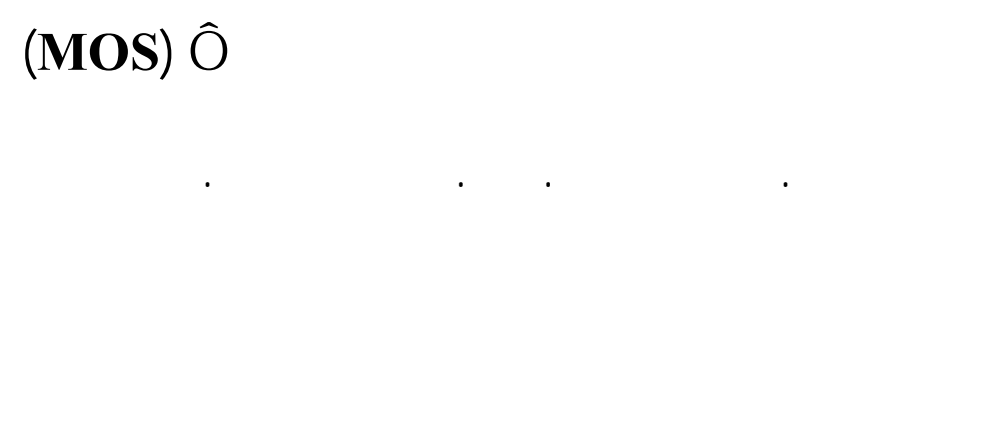

:ď

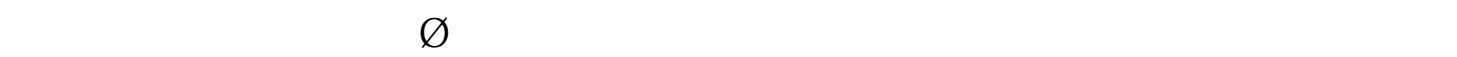
SiO2 tfūo Eh

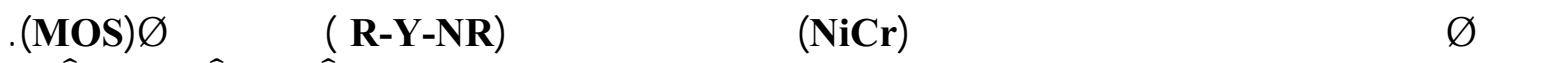
ŝว

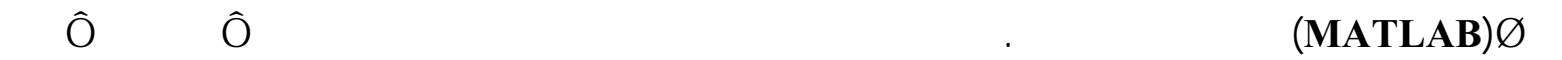

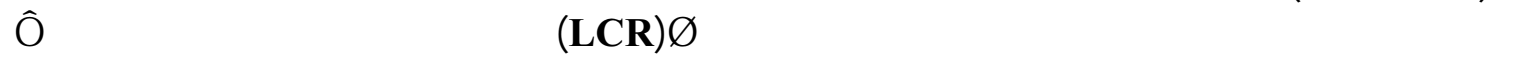

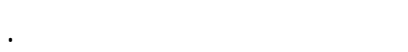

Received $24^{\text {th }}$ Sep. 2003

Accepted $6^{\text {th }}$ Jun 2005 


\section{$\begin{array}{llll}\text { Al- Rafidain Engineering } & \text { Vol. } 14 & \text { No. } 1 & 2006\end{array}$}

\section{1- INTRODUCTION}

In the recent years, there have been rapidly growing interest and activity in thin film integrated circuits as an approach to microelectronics. Electronic circuits have been fabricated on the basis of replacing conventional lumped elements with their thin film equivalents.

The majority of the up-dated work, however has been concerned with the investigation of sandwiched three layer rectangular and exponential shaped structures. In these structures, alternate layers of resistive and dielectric films are deposited on appropriate substrates to form four terminal R-Y-NR network [1].

The method of analysis that was used to obtain the steady state ac response and the response to a unit step is rather straightforward. It is shown that the partial differential equation relating voltage, position, and time is of second order homogeneous ordinary linear differential equation [2]. If the gate of the MOS structure is deposited as a strip of resistor film like NiCr, the MOS structure can be analyzed as R-Y-NR network [3].

\section{2- Open circuit voltage transfer function of exponential DP R-Y-NR subnetwork}

The matrix parameter functions (MPFs) of a solvable DP R-Y-NR network are defined with the following symbols [2]:

$$
\begin{aligned}
& r=\left|\begin{array}{ll}
M_{0}^{\prime} & F_{0}^{\prime} \\
M_{L}^{\prime} & F_{L}^{\prime}
\end{array}\right| \\
& g=(1+N) R_{0}\left|\begin{array}{ll}
M_{0}^{\prime} & F_{0}^{\prime} \\
M_{L}^{\prime} & F_{L}^{\prime}
\end{array}\right| \\
& b=(1+N) R_{L}\left|\begin{array}{ll}
M_{0}^{\prime} & F_{0}^{\prime} \\
M_{0} & F_{0}
\end{array}\right| \\
& a=(1+N) R_{0}\left|\begin{array}{ll}
M_{L}^{\prime} & F_{L} \\
M_{L} & F_{L}
\end{array}\right| \\
& h=(1+N) R_{L}\left|\begin{array}{ll}
M_{0}^{\prime} & F_{0} \\
M_{L} & F_{L}
\end{array}\right| \\
& y=(1+N)^{2} R_{0} R_{L}\left|\begin{array}{ll}
M_{L} & F_{L} \\
M_{0} & F_{0}
\end{array}\right|
\end{aligned}
$$

Employing the technique of subnetwork generation [4,5], the open circuit voltage transfer function $\mathrm{T}_{\mathrm{vo}}$ of the exponential distributed parameter two-port three layer subnetworks of Fig. (1) is obtainable in terms of the matrix parameter functions (MPFs). 
The exponential distributed parameter R-Y-NR structure consists of two resistive layers with per unit length (PUL) series resistance $R=R_{0} \exp (K x)$ and $N R=N R_{0} \exp (K x)$ for the first and second resistive layers respectively.

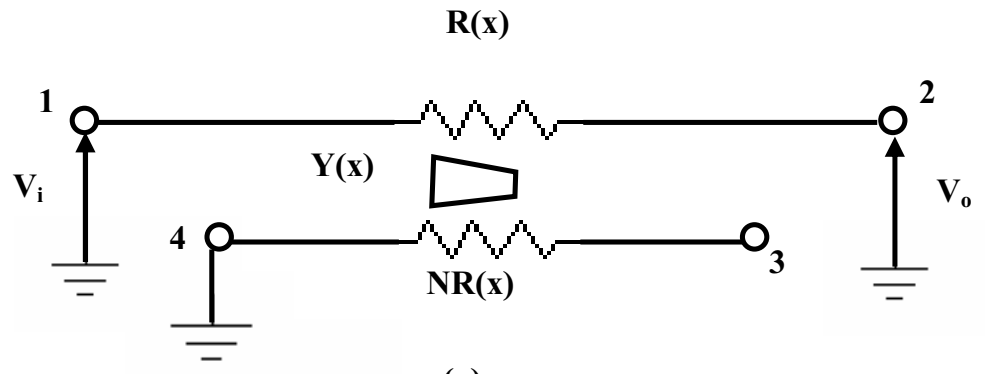

(a)

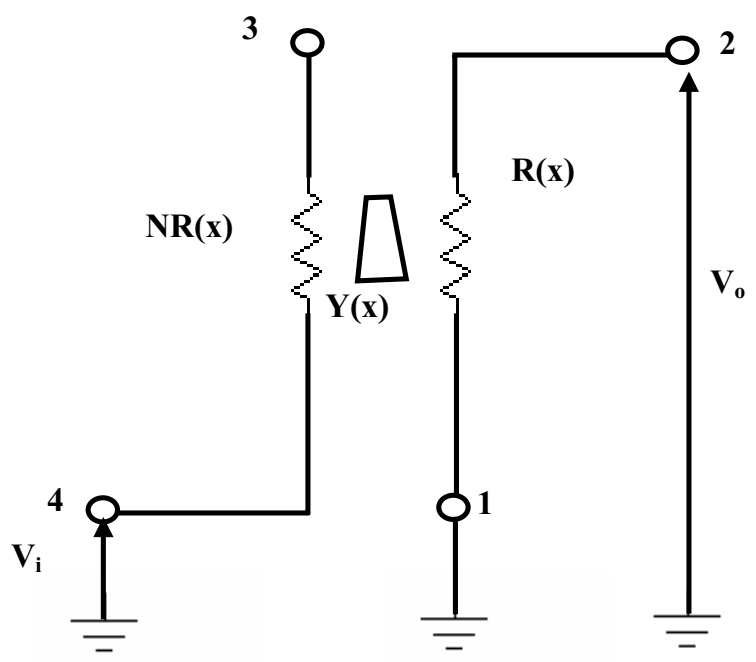

(b)

Fig.(1) (a) IOFG (1-Input, 2-Output, 3-Floating, 4-Ground) configuration (b) GOFI (1-Ground, 2-Output, 3-Floating, 4-Input) configuration

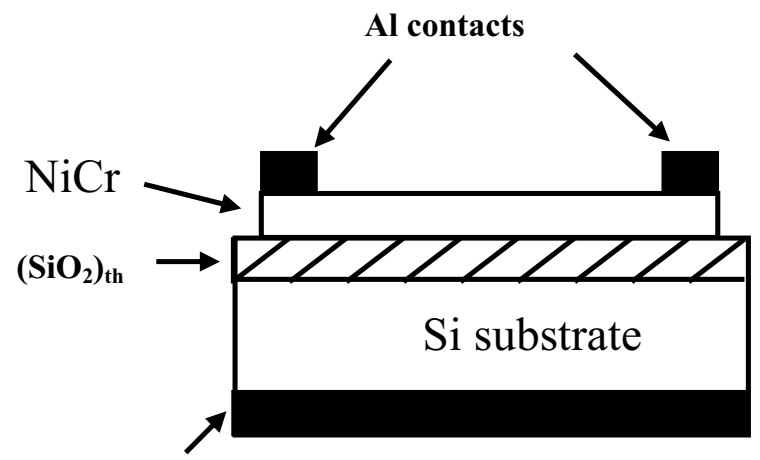

Al back contacts

Fig.(1c) Cross section of MOS structure with strip NiCr gate contact. 
These two resistive layers are separated from each other by an intermediate dielectric layer for which the per unit length (PUL) shunt capacitance is $\mathrm{C}=\mathrm{C}_{0} \exp (-\mathrm{Kx})$ and shunt conductance is $\mathrm{G}=\mathrm{G}_{0} \exp (-\mathrm{Kx})$ where $\mathrm{N}$ is a dimensionless constant

representing the a ratio of the two resistive layers, $R_{0}$ is a PUL resistive constant, $C_{0}$ is a PUL capacitive constant, $\mathrm{G}_{\mathrm{o}}$ is a PUL conductive constant and

$\mathrm{K}$ is a PUL exponential taper constant. The open circuit voltage transfer function [4] for the Subnetwork in Fig. (1a) is:

$$
\mathrm{T}_{\mathrm{V}_{\mathrm{O}}}=\frac{\mathrm{V}_{\mathrm{O}}}{\mathrm{V}_{\mathrm{i}}}=\frac{\mathrm{a}+\mathrm{Ng}}{(1+\mathrm{N})_{\mathrm{g}}}
$$

and that for the subnetwork in Fig.(1b) is:

$$
\mathrm{T}_{\mathrm{V}_{\mathrm{o}}}=\frac{\mathrm{v}_{\mathrm{O}}}{\mathrm{v}_{\mathrm{i}}}=\frac{\mathrm{g}-\mathrm{a}}{(1+\mathrm{N}) \mathrm{g}}
$$

where $g$ and a are (MPFs) for the exponential distributed parameter (DP) R-Y-NR structure. For structure of length $L$ and ac signal, they are identified as [5]:

$$
\begin{aligned}
& g=\cosh (m L)+\frac{K}{2} \sinh (m L) \\
& a=m \exp \left(\frac{K L}{2}\right)^{2} \\
& m=\sqrt{(K / 2)^{2}+\left(j \omega C_{o}+G_{o}\right) R_{o}(1+N)} \\
& \omega=\text { angular frequency }=2 \pi \mathrm{f}
\end{aligned}
$$

For $\mathrm{N}=0$ which means that the second resistive layer is perfect conductive film, Equations (7) and (8) will respectively be abbreviated to:

$$
\begin{array}{ll}
\frac{V_{o}}{V_{i}}=\frac{a}{g} & \text { from Fig.(1a) } \\
\frac{V_{o}}{V_{i}}=\frac{g-a}{g}=1-\frac{a}{g} & \text { from Fig.(1b) }
\end{array}
$$

Substituting the matrix parameter functions in the Equations (11) and (12) will respectively give:

$$
\frac{V_{o}}{V_{i}}=\frac{m \exp \left(\frac{K L}{2}\right)}{m \cosh (m L)+\frac{K}{2} \sinh (m L)} \quad \text { from Fig.(1a) }
$$




$$
\frac{V_{O}}{V_{i}}=1-\frac{m \exp \left(\frac{K L}{2}\right)}{m \cosh (m L)+\frac{K}{2} \sinh (m L)} \quad \text { from Fig.(1b) }
$$

Considering the uniform distributed thin film R-Y-NR network; that means the constant of exponential taper is zero $(\mathrm{K}=0)$, and substituting in the Equations (13) and (14) leads respectively to get:

$$
\begin{array}{ll}
\frac{V_{O}}{V_{i}}=\frac{m}{m \cosh (m L)}=\frac{1}{\cos h(m L)}=\operatorname{Sech}(m L) \quad \text { from Fig. (1a) } \\
\frac{V_{O}}{V_{i}}=1-\operatorname{Sech}(m L) \quad \text { from Fig. (1b) }
\end{array}
$$

where $\mathrm{m}$ is a complex angle per unit length and

$$
m=\sqrt{j \omega C_{0} R_{0}+R_{0} G_{0}}
$$

Then the complex angle is $\mathrm{mL}=\mathrm{m} \times \mathrm{L}$ and

$$
m L=\sqrt{j \omega C_{0} R_{0} L^{2}+R_{0} G_{0} L^{2}}
$$

Let $\frac{v_{0}}{v_{i}}=T_{v 1}$ for circuit connection in Fig.(1a) and $\frac{v_{0}}{v_{i}}=T_{v 2}$ for that in Fig.(1b), then:

$$
T_{v 1}=\operatorname{Sech}(m L)
$$

and $T_{v 2}=1-\operatorname{Sech}(m L)$

Subtracting (20) from (19) and manipulating the results lead to:

$m L=\operatorname{Sech}^{-1}\left(\frac{T_{v 1}+1-T_{v 2}}{2}\right)$

And hence:

$$
(m L)^{2}=\left[\operatorname{Sech}^{-1}\left(\frac{T_{v 1}+1-T_{v 2}}{2}\right)\right]^{2}
$$

From Equation (18):

$(m L)^{2}=\mathrm{j} \omega \mathrm{C}_{\mathrm{o}} \mathrm{R}_{\mathrm{o}} \mathrm{L}^{2}+\mathrm{R}_{\mathrm{o}} \mathrm{G}_{\mathrm{o}} \mathrm{L}^{2}$

Joining Equations (21) and (22) gives: 
$\mathrm{C}=C_{O} L=\frac{\operatorname{Im}\left[\operatorname{Sech}^{-1}\left(\frac{T_{v 1}+1-T_{v 2}}{2}\right)\right]^{2}}{\omega R_{O} L}$

$\mathrm{G}=G_{o} L=\frac{\operatorname{Re}\left[\operatorname{Sech}^{-1}\left(\frac{T_{v 1}+1-T_{v 2}}{2}\right)\right]^{2}}{R_{o} L}$

\section{3- EXPERIMENTAL RESULTS}

For the sake of showing the accuracy of the proposed method, the shunt capacitance and shunt conductance measurements have been carried out on a certain MOS samples. These samples are accomplished by depositing a strip of $\mathrm{NiCr}$ resistor thin film as a gate contact and then depositing two dot aluminum points at the two ends of the strip for measurement purposes (see Fig.(1c)).

At the beginning, the transfer function of the device has been measured for both configurations shown in Fig.(1). The response of transfer function magnitude and its phase with respect to frequency have been plotted as shown in Figs.(2) and (3) respectively for positive gate biasing. For negative biasing, the transfer function magnitude and phase responses have been plotted as shown in Figs.(4) and (5) respectively. Matlab program has been used to compute shunt capacitance and shunt conductance for strip gate MOS structure at different frequencies. For a zero bias, the shunt capacitance and shunt conductance of the MOS structure at different frequencies have been computed. These results and those obtained using LCR meter method [6] have been plotted. As shown in Figs.(6) and (7), the results obtained from the two methods coincided with each other.

\section{4- CONCLUSIONS}

In this research the high frequency $\mathrm{C}-\mathrm{V}$ and $\mathrm{G}-\mathrm{V}$ device measurements were fulfilled using MOS structure as a thin film distributed R-Y-NR structure with four terminal two port network. This conclusion encourage using the proposed method as a tool for $\mathrm{C}-\mathrm{V}$ and $\mathrm{G}-\mathrm{V}$ plots at any frequency.

\section{5- REFERENCES}

[1] B. B. Woo, and J. M. Bartlemay, "Characteristics and applications of a tapered, thin film distributed parameter structure", IEEE Int. Convent. Rec., 11(2), PP. 56-75, 1963.

[2] K. U. Ahmed, "The two port four terminal matrix parameter functions of solvable distributed parameter Z-Y-KZ network", IEEE Trans. on circuit Theory, Vol. CT-19 (5), PP. 506-508, 1972.

[3] P. L. Swart and C. K. Campbell, "Effect of losses and parasitic on a voltage-controlled tunable distributed RC notch filter" IEEE J. Solid-State Circuits, Vol. SC-8, No. 1, PP. 35-36, 1973. 
[4] K. U. Ahmed, "Two port sub-networks of exponential distributed parameter Z-Y-KZ and Y-Z-KY micro-circuits with similar transfer functions", Microelectron Reliab, Vol. 21, No. 2, PP. 235-239, 1981.

[5] K. U. Ahmed, "A new band-reject filter configuration of three-layer thin-film exponential R-C-KR", Microelectron Reliab, Vol. 21, No. 2, PP. 241-242, 1981.

[6] P. Olivo, T. N. Nguyen, and B. Ricco, "High-field induced degradation in ultra-thin $\mathrm{SiO}_{2}$ films" IEEE Trans. on Electron Devices, Vol. 35, No. 12, PP. 2259-2267, 1988.

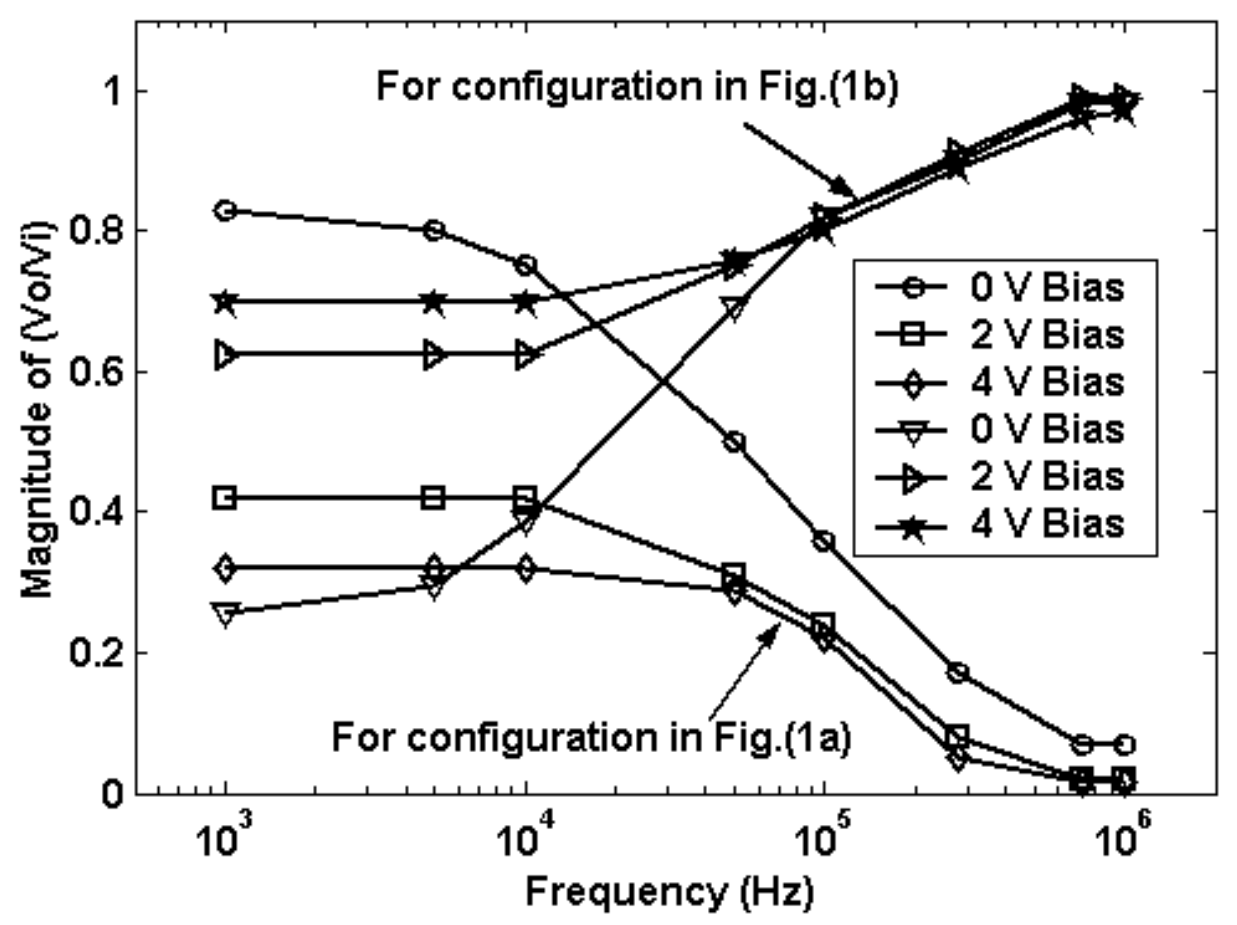

Fig.(2) Transfer function magnitude frequency response of a strip gate MOS device for different positive biases. 


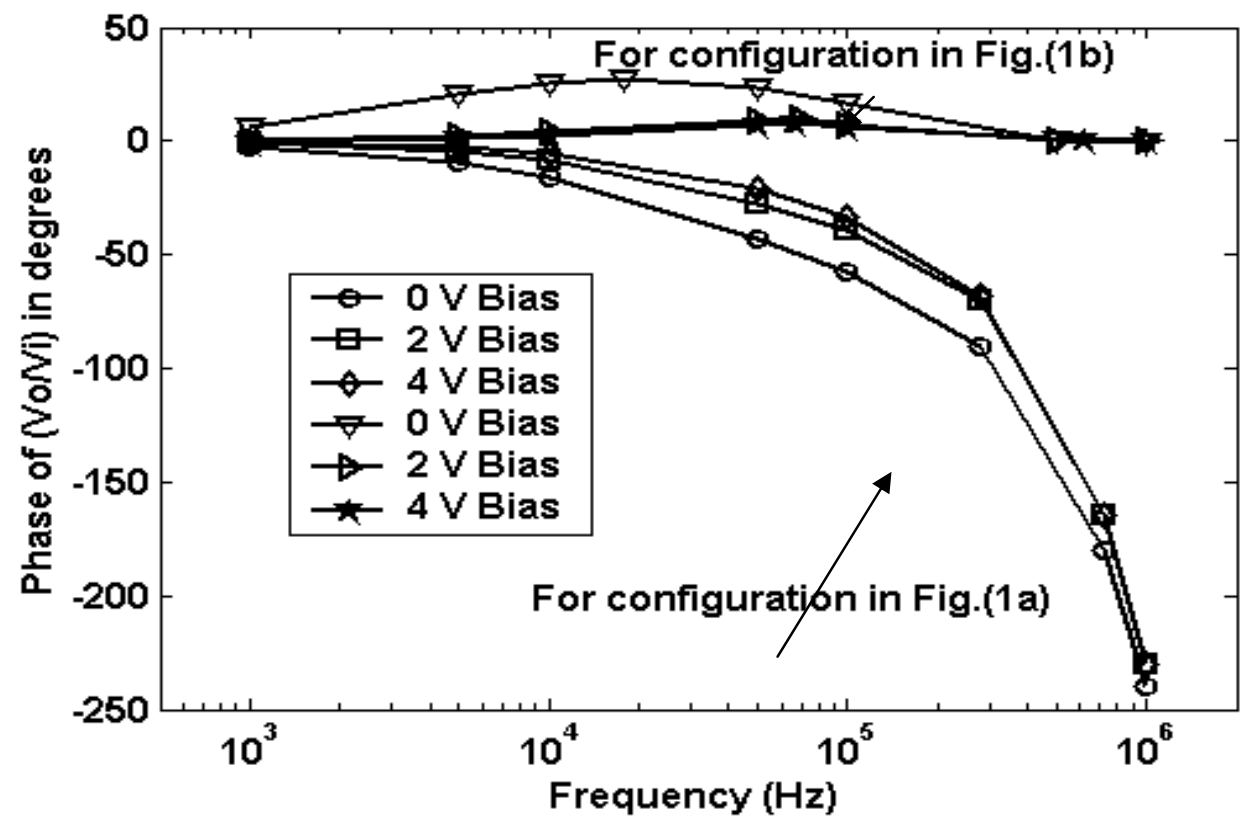

Fig.(3) Transfer function phase frequency response of a strip gate MOS device for different positive biases. 
Mohamad: New Analysis to Measure the Capacitance and Conductance

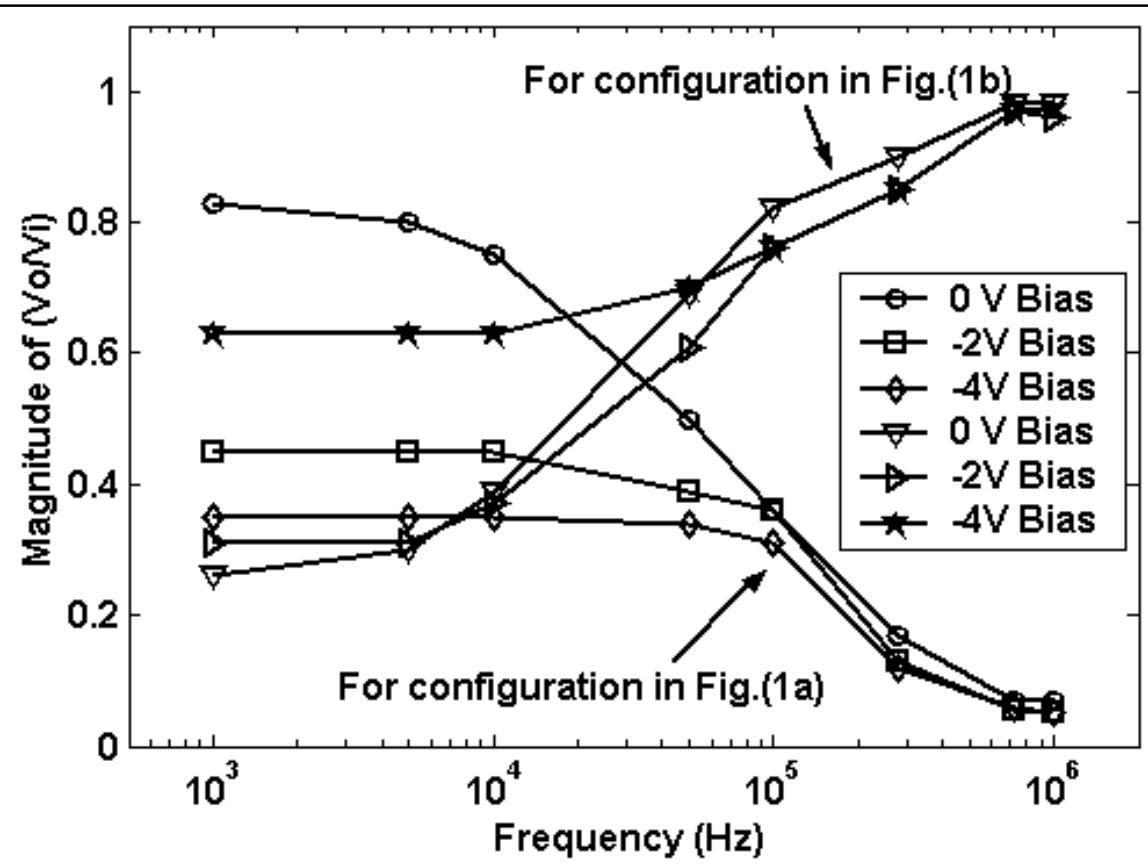

Fig.(4) Transfer function magnitude frequency response of a strip gate MOS device for different negative biases

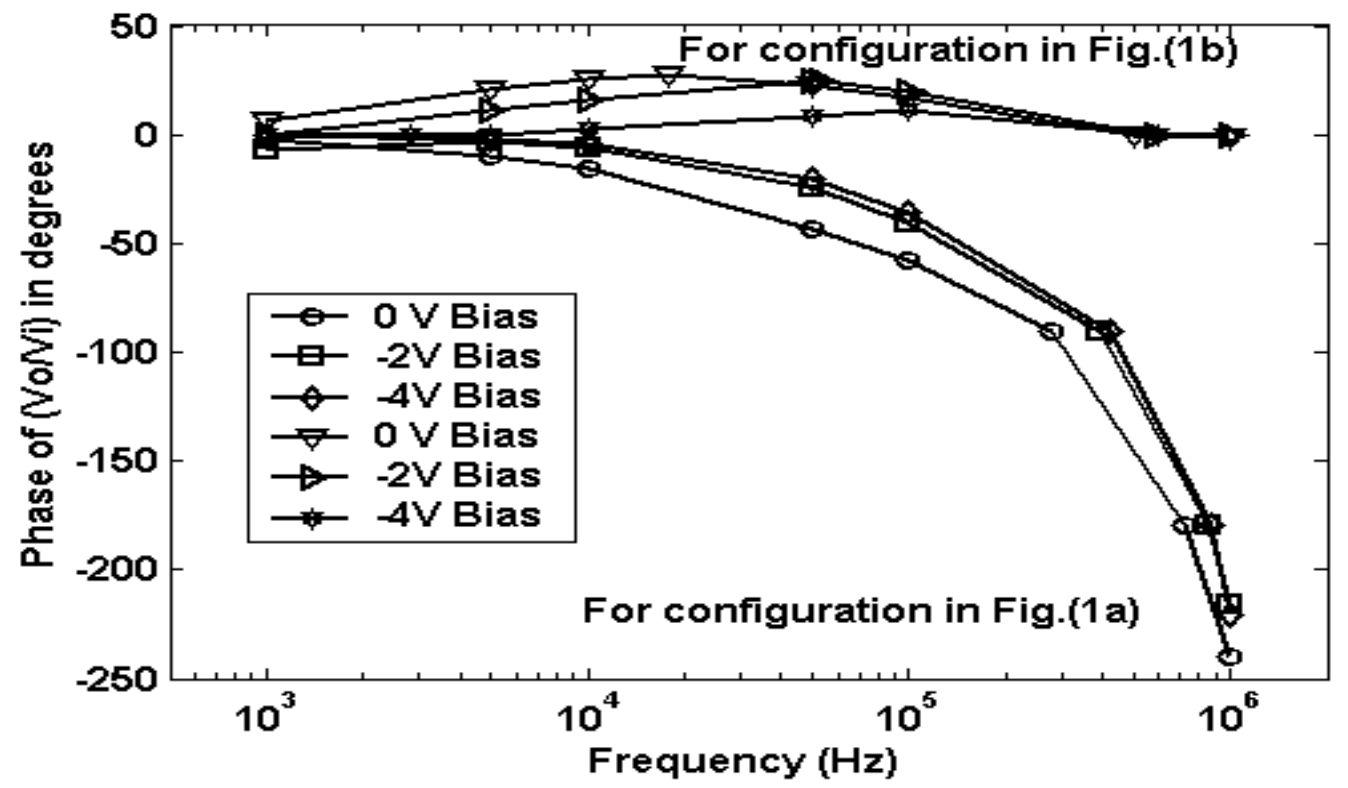

Fig.(5) Transfer function phase frequency response of a strip gate Mos device for different negative biases. 


\begin{tabular}{llll} 
Al- Rafidain Engineering & Vol. 14 & No. 1 & 2006 \\
\hline
\end{tabular}

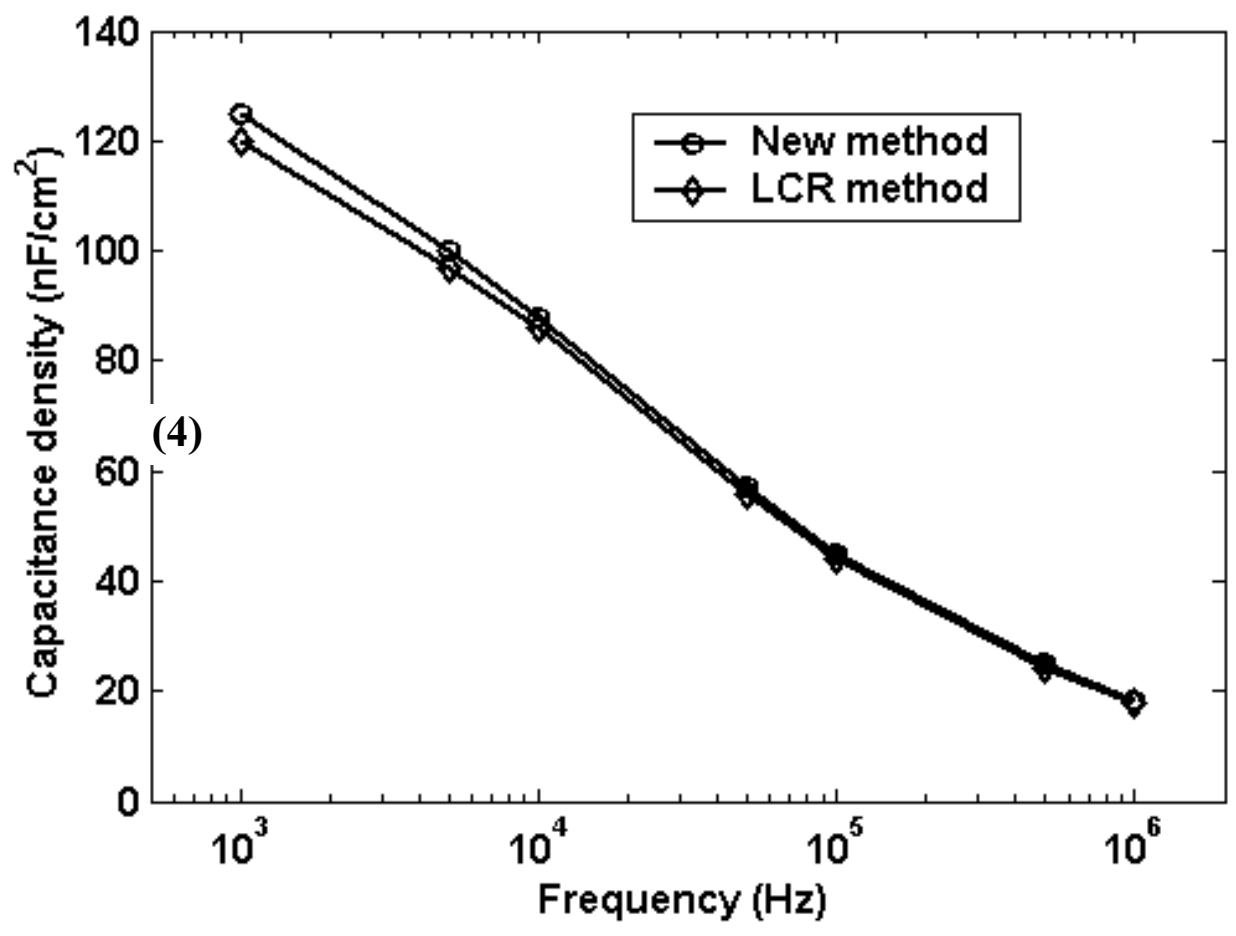

Fig.(6) Comparison between capacitance determined by the two methods for zero bias 
Mohamad: New Analysis to Measure the Capacitance and Conductance.....

Numerical Study of Free Convection Heat Transfer

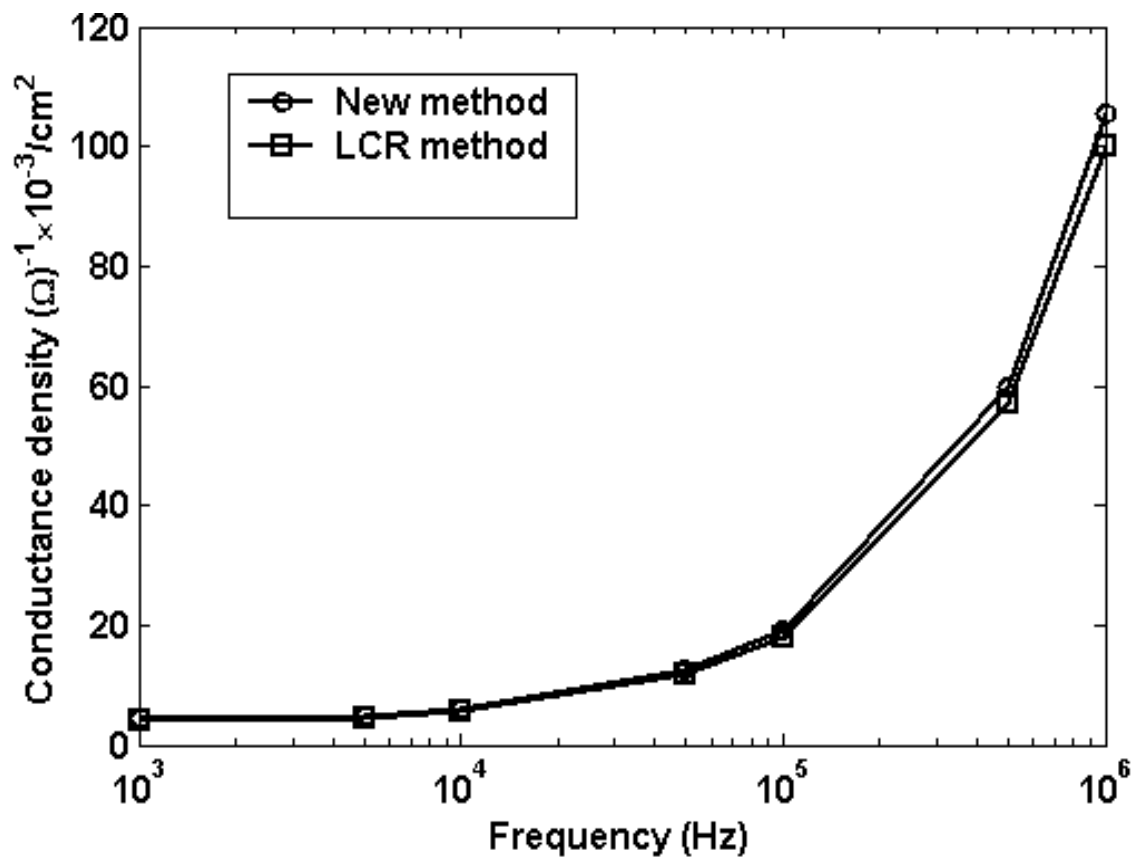

Fig.(7) Comparison between leakage conductance dedetermined by the two methods for zero bias 